\title{
One-stage surgery in combination with thoracic endovascular grafting and resection of T4 lung cancer invading the thoracic aorta and spine
}

\author{
Seijiro Sato ${ }^{1}$, Tatsuya Goto ${ }^{1}$, Terumoto Koike ${ }^{1}$, Takeshi Okamoto ${ }^{1}$, Hirokazu Shoji ${ }^{2}$, Masayuki Ohashi ${ }^{2}$, \\ Kei Watanabe ${ }^{2}$, Masanori Tsuchida ${ }^{1}$ \\ ${ }^{1}$ Division of Thoracic and Cardiovascular Surgery, ${ }^{2}$ Division of Orthopedic Surgery, Department of Regenerative and Transplant Medicine, Niigata \\ University Graduate School of Medical and Dental Sciences, Niigata, Japan \\ Correspondence to: Masanori Tsuchida. Division of Thoracic and Cardiovascular Surgery, Niigata University Graduate School of Medical and Dental \\ Sciences, 1-757 Asahimachi-dori, Chuo-ku, Niigata City, Niigata 951-8510, Japan. Email: masatsu@med.niigata-u.ac.jp.
}

\begin{abstract}
A novel strategy of one-stage surgery in combination with thoracic endovascular grafting and resection for T4 lung cancer invading the thoracic aorta and spine is described. A 56-year-old man with locally advanced lung cancer infiltrating the aortic wall and spine underwent neoadjuvant chemotherapy and thoracic irradiation, followed by en bloc resection of the aortic wall and spine with thoracic endovascular grafting. He developed postoperative chylothorax, but there were no stent graft-related events. After 3 months, computed tomography (CT) did not show aortic stent graft stenosis, migration, or deformation.
\end{abstract}

Keywords: Lung cancer; endovascular stent graft; spondylectomy; one-stage procedure

Submitted Jun 01, 2017. Accepted for publication Oct 11, 2017.

doi: $10.21037 /$ jtd.2017.10.101

View this article at: http://dx.doi.org/10.21037/jtd.2017.10.101

\section{Introduction}

Resection of locally invasive T4 lung cancer is a challenging procedure and is rarely done, especially when the tumor invades both the spine and the thoracic aorta. With the advent of endovascular therapy with thoracic endovascular aortic repair (TEVAR) in recent years, resection of the aorta and its adjacent organs has become possible. A novel strategy of one-stage surgery in combination with thoracic endovascular grafting and resection for T4 lung cancer invading the thoracic aorta and spine is presented.

\section{Case presentation}

A 56-year-old man with a smoking history of 58.5 packyears presented with back pain. His medical history was unremarkable.

Computed tomography (CT) of the chest showed a mass on the left upper lobe with probable invasion of the adjacent distal aortic arch and spine (Figure 1A). A biopsy specimen obtained by bronchoscopy showed poorly differentiated adenocarcinoma or large cell carcinoma. Magnetic resonance imaging demonstrated tumor infiltration of the T3 to T5 vertebral bodies. Positron emission tomography (PET)-CT scan showed increased metabolism in the mass with a maximum standardized uptake value (SUVmax) of 65.0, but there was no mediastinal disease or extrathoracic metastases (Figure 1B). Given these findings, the diagnosis was stage IIIA (cT4N0M0) lung cancer, and the patient was given neoadjuvant chemotherapy with 3 cycles of cisplatin-vinorelbine and 60-Gy thoracic irradiation. The patient had stopped smoking 1 month before neoadjuvant chemoradiotherapy.

At 4 weeks after chemoradiotherapy, restaging with CT and PET-CT imaging showed partial response with the SUVmax at 3.7 and without nodal and distant metastases (Figure 1C). It was then decided to perform a left upper lobectomy combined with resection of the infiltrated aortic wall and the T3 to T5 vertebral bodies, as well as posterior spinal instrumentation from C7 to T8. Cardiopulmonary bypass $(\mathrm{CPB})$ with full heparinization was considered 

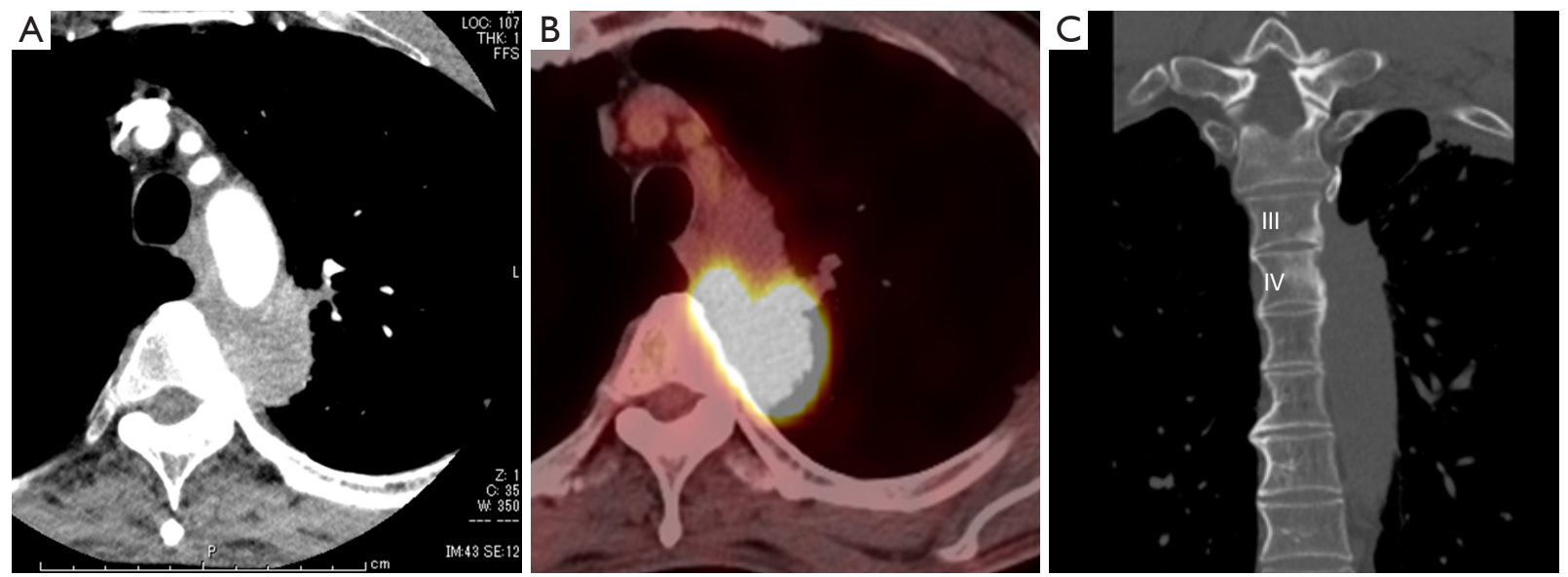

Figure 1 Preoperative images. (A) CT shows a mass of the left upper lobe invading the distal aortic wall and the $4^{\text {th }}$ thoracic vertebra; (B) PET-CT before neoadjuvant chemoradiotherapy shows increased metabolic activity in the mass; (C) CT coronal image shows osteosclerotic changes in the $3^{\text {rd }}$ and $4^{\text {th }}$ thoracic vertebrae, which strongly suggest direct tumor invasion.

to have too high morbidity and mortality risks during resection of the infiltrated aortic wall with concomitant spine resection. To avoid such risks, placement of a thoracic endovascular stent graft for the aortic invasion was planned without CPB. Before endovascular stent grafting, CT angiography was performed from the supra-aortic vessels to the common femoral arteries to assess vessel size and anatomy and to delineate the maximum possible area of aortic invasion. This surgical procedure was approved by the institutional review board of Niigata University Hospital (No. 2658), and the patient's informed consent was obtained.

The surgical procedure included four steps. First, with the patient in the supine position and after confirming the infiltrated aortic wall by palpation with forceps and marking the proximal and distal ends of the tumor with surgical clips to guide endovascular stent grafting by exploratory thoracoscopy, a $30 \mathrm{~mm} \times 95 \mathrm{~mm}$ thoracic endovascular stent graft (RELAY Plus delivery system; Bolton Medical Inc., Sunrise, FL, USA) was placed distal to the left subclavian artery to cover the infiltrated lesion while keeping a safe proximal 4-cm margin of healthy aorta. However, the distal margin of the healthy aorta was only $1.5 \mathrm{~cm}$; therefore, another thoracic endovascular stent graft (30 mm $\times 150 \mathrm{~mm}$; RELAY Plus delivery system) was placed to ensure a sufficient distal margin (Figure $2 A$ ). The second step was left upper lobectomy with en bloc resection of aortic adventitia and media, about $4 \mathrm{~cm}$ in length or $1 / 4$ of the aortic circumference, followed by dissection of the lateral aspect of the vertebral bodies and the third to fifth ribs, keeping a $3-\mathrm{cm}$ margin from tumor, with the patient in the right lateral position. Third, after lung resection, the patient was placed in the prone position, and total removal of the posterior elements (T3 to T5) was performed with bilateral transection of the T3, T4, and T6 nerve roots; dissection around the vertebral bodies (T3 to $\mathrm{T} 5$ ) and posterior spinal instrumentation from C7 to T8 using pedicle screws (Figure 2B). Finally, in the right lateral position, the surgical specimen involving the attached tumor, lung, aortic wall, chest wall, and vertebral bodies (T3 to T5) was translated forward, rotated around the spinal cord, and extracted en bloc (Figure 2C). After total removal of the affected spine, vertebral body reconstruction was performed using an expandable titanium cage. Then, in a one-stage procedure, the chest wall was reconstructed with a polytetrafluoroethylene sheet (GoreTex; WL Gore \& Associates, Flagstaff, AZ, USA), and the aortic wall defect was reinforced and the bronchial stump and polytetrafluoroethylene sheet were covered with the latissimus dorsi muscle. There were no intraoperative complications.

The patient developed postoperative chylothorax and was treated by no oral intake and pleurodesis, but there were no stent graft-related events. The final pathology confirmed ypT0N0 disease. After 3 months, repeat CT did not show aortic stent graft stenosis, migration, or deformation (Figure 2D). The patient is well, with no evidence of recurrent disease at 6 months after surgery. 

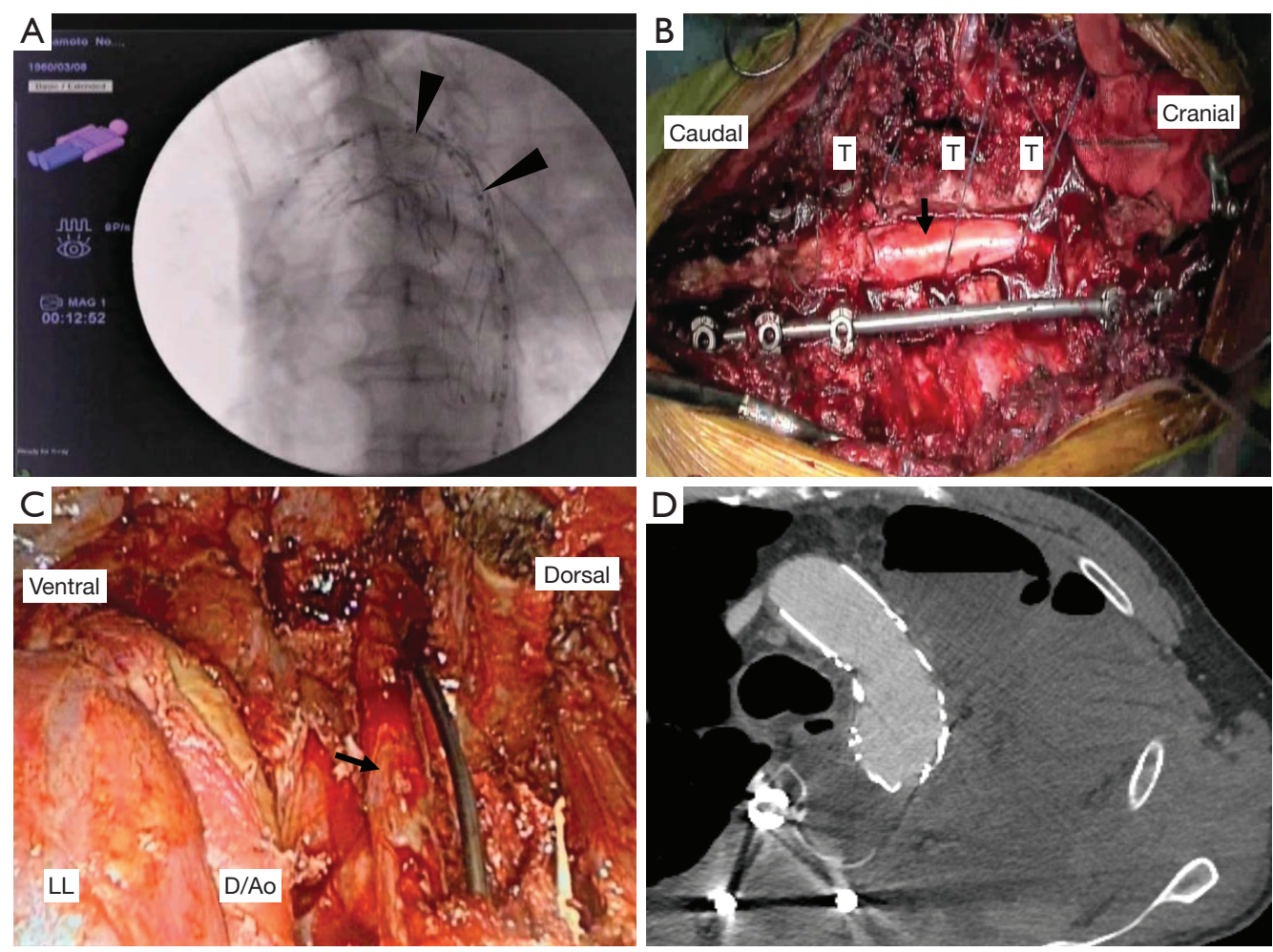

Figure 2 Intraoperative views and postoperative images. (A) Thoracic endovascular stent grafts are placed with 4-cm margins of healthy aorta beyond the proximal and distal ends of the tumor, as indicated by clips (arrowheads); (B) this view shows total removal of the posterior elements (T3 to T5), transection of the bilateral T3, T4, and T6 nerve roots, dissection around the T3 to T5 vertebral bodies, and posterior spinal instrumentation from C7 to T8 using pedicle screws in the prone position. The arrow indicates the dura mater; (C) overall intraoperative view. The arrow indicates the dura mater; (D) at 3 months after surgery, CT shows no stent graft-related complications. D/Ao, descending aorta; LL, lower lob.

\section{Discussion}

Surgical resection of a T4 lung cancer infiltrating the spine and the aorta is a challenging procedure. The evolution of spinal stabilization techniques has facilitated successful and safe vertebral body resection (1-3). Regarding aortic invasion, Marulli et al. first reported in 2008 the procedure of resection of lung metastases invading the aortic wall using an endovascular stent graft without CPB (4). Recently, aortic wall resection under TEVAR has been reported as a less invasive technique. In most cases, the procedure is sequential, with deployment of the thoracic aortic stent graft several days before lung cancer surgery $(5,6)$. Collaud et al. reported that a staged procedure for aortic endografting and resection allowed clinical and radiologic assessment of the complications of stent grafting (6). Recently, Marulli et al. reported that a one-stage procedure was possible without any problems (7) and noted two potential advantages of the one-stage approach. One is avoidance of additional anesthesia and the other, which was more relevant, is avoidance of a useless procedure in cases that do not have deep aortic invasion during thoracotomy.

At our institution, we have previously performed three cases of one-stage procedures for advanced T4 lung cancer infiltrating the aortic wall using endovascular stent grafts. The average duration of endovascular stent graft placement was 98 minutes (range, 49-149 minutes). To avoid migration after endovascular stent graft replacement, we selected a stent graft that was about $10 \%$ larger than the native aorta and occasionally performed ballooning after deployment. For reinforcement of the aortic wall defect, in cases of aortic resection without spinal surgery, using a bioprosthetic such as bovine pericardium or alloderm, which could be cut to size and avoid graft site harvest morbidity, might be helpful for reinforcement of the aortic wall defect. For the most part, we have not experienced any serious perioperative complications. 
Therefore, we deemed the one-stage procedure for aortic wall invasion to be appropriate. On the other hand, the duration of surgery for lung cancer invading the spine and aortic wall was longer. Similar to additional anesthesia, this prolonged surgery can be considered to add to the invasiveness of the procedure. However, along with the progress in spinal surgery, a one-stage approach for this advanced disease is acceptable for selected patients. In this case, we learned that the patient turned out to be ypT0N0 after the resection. Given that, it was impossible to make a precise assessment about whether the malignant cells were present preoperatively, which might be a problem to consider.

To the best of our knowledge, this patient is the first reported case of aortic resection using endovascular stent grafting and spondylectomy in one stage. However, we emphasize that this procedure should be undertaken by a multidisciplinary team of thoracic, vascular, and spinal surgeons that can provide the necessary perioperative management of possible immediate complications.

\section{Acknowledgements}

None.

\section{Footnote}

Conflicts of Interest: The authors have no conflicts of interest to declare.

Informed Consent: Written informed consent was obtained from the patient for the publication of this case report and any accompanying images.

\section{References}

1. Anraku M, Waddell TK, de Perrot M, et al. Induction chemoradiotherapy facilitates radical resection of T4 non-small cell lung cancer invading the spine. J Thorac Cardiovasc Surg 2009;137:441-7.e1.

2. Chen F, Takahashi A, Omasa M, et al. En bloc total vertebrectomy for lung cancer invading the spine. Lung Cancer 2008;61:137-9.

3. Yokomise H, Gotoh M, Okamoto T, et al. En bloc partial vertebrectomy for lung cancer invading the spine after induction chemoradiotherapy. Eur J Cardiothorac Surg 2007;31:788-90.

4. Marulli G, Lepidi S, Frigatti P, et al. Thoracic aorta endograft as an adjunct to resection of a locally invasive tumor: a new indication to endograft. J Vasc Surg 2008;47:868-70.

5. Berna P, Bagan P, De Dominicis F, et al. Aortic endostent followed by extended pneumonectomy for T4 lung cancer. Ann Thorac Surg 2011;91:591-3.

6. Collaud S, Waddell TK, Yasufuku K, et al. Thoracic aortic endografting facilitates the resection of tumors infiltrating the aorta. J Thorac Cardiovasc Surg 2014;147:1178-82; discussion 1182.

7. Marulli G, Rea F, Zampieri D, et al. Safe resection of the aortic wall infiltrated by lung cancer after placement of an endoluminal prosthesis. Ann Thorac Surg 2015;99:1768-73.

Cite this article as: Sato S, Goto T, Koike T, Okamoto T, Shoji H, Ohashi M, Watanabe K, Tsuchida M. One-stage surgery in combination with thoracic endovascular grafting and resection of T4 lung cancer invading the thoracic aorta and spine. J Thorac Dis 2017;9(11):E1009-E1012. doi: 10.21037/ jtd.2017.10.101 